\title{
Performance Analysis for Optimizing Threshold Level Control of a Receiver in Asynchronous 2.5 Gbps/1.2 Gbps Optical Subscriber Network with Inverse Return to Zero(RZ) Coded Downstream and NRZ Upstream Re-modulation
}

\author{
SangJo Park* \\ Department of Information and Communications, Seowon University, 231 Musimseoro, \\ Heungduk-gu, Cheongju 361-742, Korea \\ Bong Kyu Kim \\ Biosensor Research Team, ETRI, 138 Gajeongno, Yuseong-gu, Daejeon 305-700, Korea
}

(Received May 11, 2009 : revised July 7, 2009 : accepted July 8, 2009)

\begin{abstract}
We propose the performance enhancing method optimization of an asynchronous 2.5 Gbps/1.25 Gbps optical subscriber network with inverse RZ (Return to Zero) coded downstream and NRZ (Non Return to Zero) upstream re-modulation by adjusting threshold level control of a receiver. We theoretically analyze the BER (Bit Error Rate) performance by modeling the occurrence of BER by simulation with MATLAB according to the types of downstream data. The results have shown that the normalized threshold level in an optical receiver could be saturated at $1 / 3$ as the SNR (Signal to Noise Ratio) increases. The needed SNR for obtaining the BER $10^{-9}$ can be reduced by $\sim 5 \mathrm{~dB}$ by optimizing the normalized threshold level at $1 / 3$ instead of by using the conventional receiver with threshold level of 0.5 . The proposed system can be a useful technology for asynchronous optical access networks with asymmetric upstream and downstream data rates, because the improved minimum receiving power could replace a light source with a source with lower power and lower cost in an OLT (Optical Line Termination).
\end{abstract}

Keywords : Fiber-to-the-home (FTTH), Optical subscriber network, Inverse return to zero code, Optical Network Unit (ONU), Optical Line Termination (OLT)

OCIS codes: (060.0060) Fiber optics and optical communications; (060.2380) Fiber optics sources and detectors; (060.2330) Fiber optics communications

\section{INTRODUCTION}

A present subscriber access system needs a broadband transmission link that offers interactive multimedia services such as voice, high quality video-on-demand, high speed data, and so on. Fiber-optic transmission systems such as the FTTH (Fiber-To-The-Home) have been introduced into subscriber access networks to improve the characteristics of transmission. A FTTHbased passive optical network (PON) is shown in Fig. 1, where several ONUs (Optical Network Units) are connected to an OLT (Optical Line Termination) by

*Corresponding author: parks@seowon.ac.kr using an optical fiber and a routing node (RN) [1-4].

A PON is an effective technology for an optical subscriber network because of ease of installation and low operational costs through sharing the optical fiber and an OLT. However, the conventional WDM-PON system needs complex circuits such as wavelength and power controllers. Wavelength and power controllers and light sources were removed at $\mathrm{ONU}$ in order to simplify the circuit and reduce the cost. The re-modulation scheme is one of the methods for removing an optical source at the ONU and the use of centralized light source at OLT. The re-modulation schemes reusing downstream light for upstream data transmission have been proposed and studied in optical access 


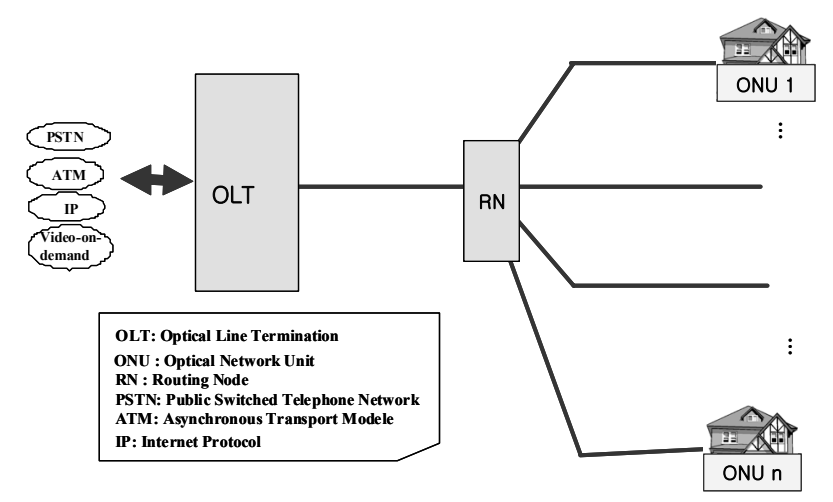

FIG. 1. FTTH-based passive optical network(PON).

networks [5-17]. There are various re-modulation schemes depending on the downstream data formats. Among many re-modulation schemes, Manchester and inverse return-to-zero (RZ) coded re-modulation schemes have been proposed in order to provide asynchronous full duplex operations [13-17].

In this paper, we propose the performance enhancing method of asynchronous $2.5 \mathrm{Gbps} / 1.25 \mathrm{Gbps}$ optical subscriber network with inverse RZ coded downstream and NRZ (Non Return to Zero) upstream re-modulation by adjusting the threshold level of a receiver. We theoretically analyze BER (Bit Error Rate) performance by modeling the occurrence of BER by simulation with MATLAB according to the types of downstream data.

\section{ASYNCHRONOUS 2.5 GBPS/1.25 GBPS OPTICAL SUBSCRIBER NETWORK WITH INVERSE RZ CODED DOWNSTREAM AND NRZ UPSTREAM RE-MODULATION}

An asynchronous 2.5 Gbps/1.25 Gbps optical subscriber network with inverse RZ coded downstream and NRZ upstream re-modulation is shown in Fig. 2. The output of a laser diode (LD) is encoded by inverse RZ code for 2.5 Gbps downstream data using an inverse RZ code generator at the OLT, where they are converted into '01' and ' 11 ' when downstream data are ' 1 ' and ' 0 ', respectively. Downstream data modulated with inverse RZ coded downstream are shown in Fig. 3. At the ONU, the encoded light is split by a 50:50 fiber coupler. One output of the coupler is connected to a photodiode (PD) and inverse RZ coded downstream data are detected. The other output is fed into a Mach-Zehnder interferometric intensity modulator (MZI) for the upstream data transmission. The latter is re-modulated by an MZI driven by a 1.25 Gbps NRZ data and transmitted back to the OLT. The re-modulated upstream data are detected at a receiver which consists of a PD, low pass filter (LPF), and a limiting amplifier

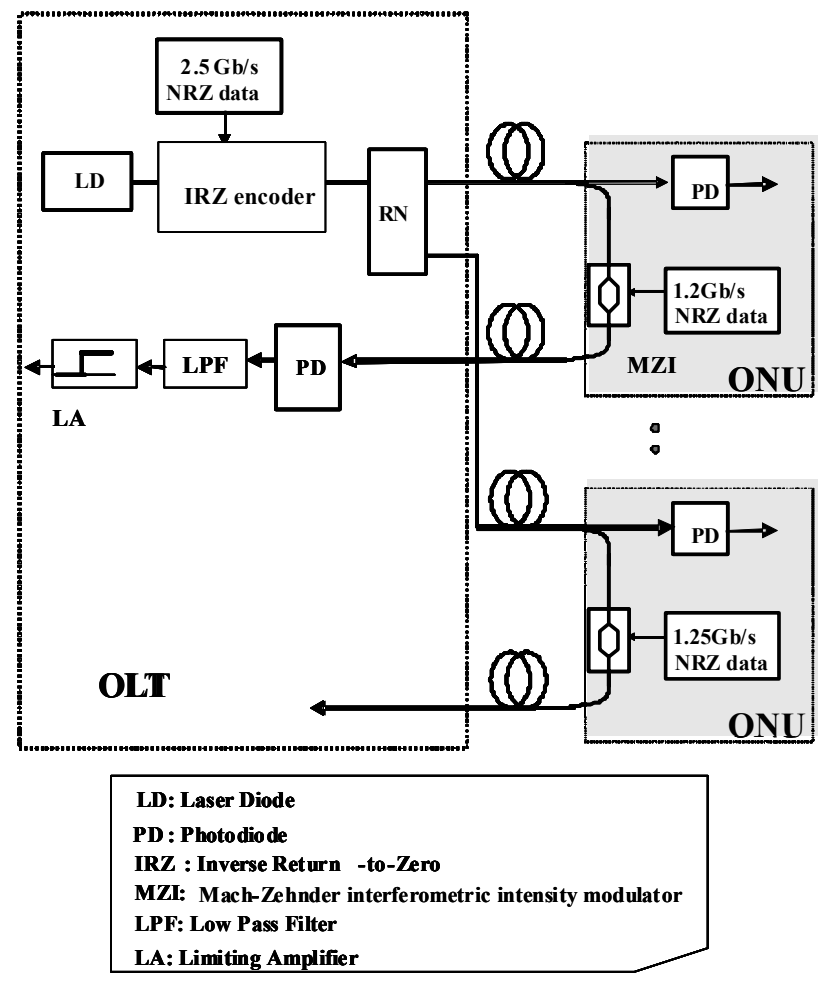

FIG. 2. An asynchronous 2.5 Gbps/1.25 Gbps optical subscriber network with inverse RZ coded downstream and NRZ upstream re-modulation.

Downstream NRZ data
$(2.5 \mathrm{~Gb} / \mathrm{s})$

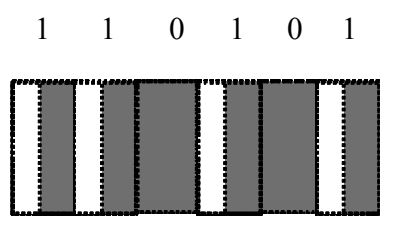

Inverse $R Z$ coded Downstream data

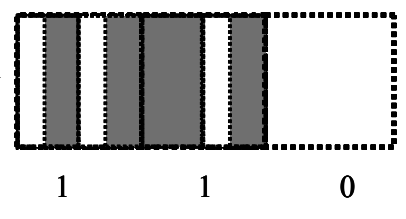

$(1.25 \mathrm{~Gb} / \mathrm{s})$

FIG. 3. Downstream data modulated with inverse RZ coded downstream.

(LA). The threshold level of the limiting amplifier is controlled by adjusting the offset voltage.

We analyze NRZ upstream re-modulation data modulated by inverse RZ coded downstream by dividing one period of $1.25 \mathrm{Gbps}$ into four sectors. Two bits of upstream data exist during one bit of downstream data. As downstream inverse RZ coded signals are '01' and ' 11 ' for data bits of ' 1 ' and ' 0 ', one pulse and two pulses exist in one bit of upstream data, respectively. When the clock synchronization between downstream and upstream data is kept, there are two, three, four 
TABLE 1. NRZ upstream re-modulated data with inverse RZ coded downstream according to three bits of downstream data

\begin{tabular}{|c|c|c|c|}
\hline Type & $\begin{array}{c}\text { downstream } \\
\text { 3bits A B C }\end{array}$ & Probability & $\begin{array}{l}\text { No. of pulses in bit } \\
\text { ' } 1 \text { ' of upstream }\end{array}$ \\
\hline $\mathrm{a}$ & 111 & $\mathrm{Pl}_{\mathrm{a}}=1 / 8$ & 2 \\
\hline $\mathrm{b}$ & $\begin{array}{lll}0 & 1 & 1 \\
1 & 1 & 0 \\
\end{array}$ & $\mathrm{Pl}_{\mathrm{b}}=1 / 4$ & $2 \sim 3$ \\
\hline $\mathrm{c}$ & $\begin{array}{lll}0 & 1 & 0 \\
1 & 0 & 1 \\
\end{array}$ & $\mathrm{Pl}_{\mathrm{c}}=1 / 4$ & 3 \\
\hline $\mathrm{d}$ & $\begin{array}{lll}0 & 0 & 1 \\
1 & 0 & 0 \\
\end{array}$ & $\mathrm{Pl}_{\mathrm{d}}=1 / 4$ & $3 \sim 4$ \\
\hline e & $\begin{array}{lll}0 & 0 & 0\end{array}$ & $\mathrm{Pl}_{\mathrm{e}}=1 / 8$ & 4 \\
\hline
\end{tabular}

pulses in one bit of upstream data according to downstream data. However clock synchronization circuits are needed to keep the clock synchronization between downstream and upstream data. We adopt asynchronous communication between downstream and upstream data for simple structure of ONU with low cost.

In this paper, we analyze BER performance and propose the performance enhancing method by controlling the threshold level of a receiver for asynchronous 2.5 Gbps/ 1.25 Gbps optical subscriber network with inverse RZ coded downstream and NRZ upstream re-modulation. When the clock synchronization between downstream and upstream data is not kept, the number of pulses is between two and four in one bit of upstream data according to downstream data. We can classify NRZ upstream re-modulated data with inverse $\mathrm{RZ}$ coded downstream according to three bits of downstream data as shown in Table 1. The number of pulses varies between two and four in one bit of upstream data according to three bits of downstream data and the probability for each case is shown in Table 1 .

\section{ANALYSIS OF BER PERFORMANCE USING MODELING OF BER OCCURRENCE}

The power component at the output of the LPF at a receiver in OLT is the sum of signal power and noise power. The noise power at the output of LPF is assumed to be a zero-mean Gaussian distributed function. Assuming that the data bits 1 and 0 are transmitted with a probability of 0.5 , the BER is given by

$$
B E R=\frac{1}{2}[P(0 / 1)+P(1 / 0)]
$$

where $P(0 / 1)$ and $P(1 / 0)$ are error probabilities when detected data are ' 0 ' and ' 1 ' for ' 1 ' and ' 0 ' of upstream

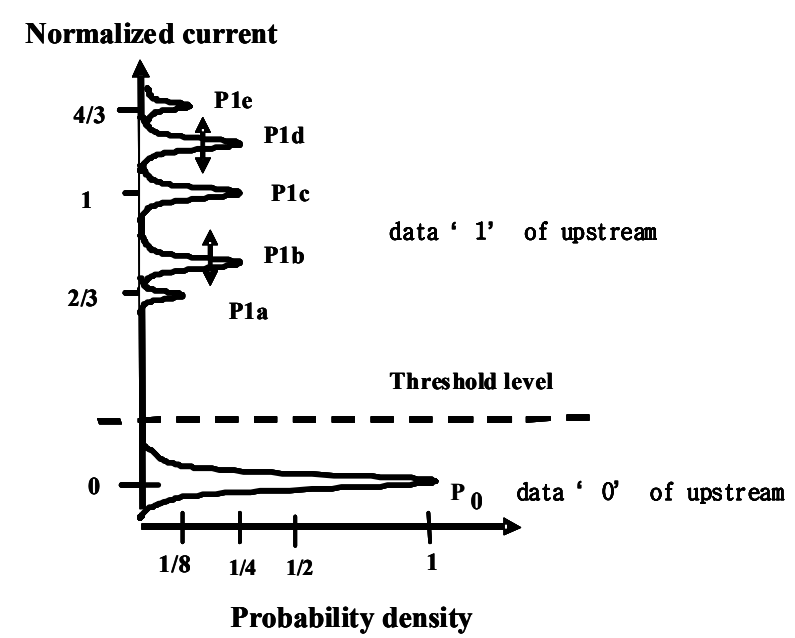

FIG. 4. Power density function of remodulated upstream with inverse RZ coded downstream according to downstream data.

data, respectively.

When upstream data is '0', the signal power is zero. So the error probability, $P(1 / 0)$ at the output of an LA is given by

$$
P(1 / 0)=\frac{1}{2} \operatorname{erfc}\left(\frac{I_{D}-I_{0}}{\sigma_{0} \sqrt{2}}\right)
$$

where $I_{0}$ and $I_{D}$ are the mean current for the data bit ' 0 ' and the threshold current, respectively, and $\sigma_{0}$ is the current standard deviation of the noise current for the data bit of ' 0 ' [18].

However, when upstream data is ' 1 ', the signal power varies according to downstream data. We consider the mean current for the data bit ' 1 ' by classifying it into five cases as shown in Table 1 . When upstream data is ' 1 ', the mean number of pulses transmitted at a receiver in OLT is three. So when the number of pulses is three, the normalized current at the output of LPF is defined as 1 . In the cases of type a, type $\mathrm{c}$ and type e defined as Table 1 , the normalized currents are $2 / 3$, 1 and $4 / 3$ and the probability densities are $P_{1 a}, P_{1 c}$, and $P_{1 e}$, respectively as shown in Fig. 4 . In the cases of type b, the normalized current varies from $2 / 3$ to 1 and the probability density is $P_{1 b}$. In the cases of type $\mathrm{d}$, the normalized current varies from 1 to $4 / 3$ and the probability density is $P_{1 d}$.

When upstream data is ' 1 ', the error probability, $P(0 / 1)$ at the output of an LA is given by

$$
P(0 / 1)=P_{a}(0 / 1)+P_{b}(0 / 1)+P_{c}(0 / 1)+P_{d}(0 / 1)+P_{e}(0 / 1)
$$

where $P_{a}(0 / 1), P_{b}(0 / 1), P_{c}(0 / 1), P_{d}(0 / 1)$ and $P_{e}(0 / 1)$ are error probabilities for type a, type b, type c, type 
$\mathrm{d}$ and type e in the case upstream data is ' 1 ', respectively. As the noise power is random variables, we can assume noise at the output of an LPF is a zero-mean Gaussian distributed function.

$P_{a}(0 / 1), P_{b}(0 / 1), P_{c}(0 / 1), P_{d}(0 / 1)$ and $P_{e}(0 / 1)$ are expressed as

$$
\begin{aligned}
& P_{a}(0 / 1)=P_{1 a} \frac{1}{2} \operatorname{erfc}\left(\frac{2 / 3-I_{D}}{\sigma_{1} \sqrt{2}}\right) \\
& P_{b}(0 / 1)=\int_{I=2 / 3}^{1} P_{1 b}^{\prime} \frac{1}{2} \operatorname{erfc}\left(\frac{I-I_{D}}{\sigma_{1} \sqrt{2}}\right) d I \\
& P_{c}(0 / 1)=P_{1 c} \frac{1}{2} \operatorname{erfc}\left(\frac{1-I_{D}}{\sigma_{1} \sqrt{2}}\right) \\
& P_{d}(0 / 1)=\int_{I=1}^{4 / 3} P_{1 d}^{\prime} \frac{1}{2} \operatorname{erfc}\left(\frac{I-I_{D}}{\sigma_{1} \sqrt{2}}\right) d I \\
& P_{e}(0 / 1)=P_{1 e} \frac{1}{2} \operatorname{erfc}\left(\frac{4 / 3-I_{D}}{\sigma_{1} \sqrt{2}}\right)
\end{aligned}
$$

where $P_{1 b}$ and $P_{1 d}$ are given by

$$
\begin{aligned}
& \int_{I=2 / 3}^{1} P_{1 b}^{\prime} d I=1 / 4 \\
& \int_{I=1}^{4 / 3} P_{1 d}^{\prime} d I=1 / 4
\end{aligned}
$$

and $\sigma_{1}$ is the current standard deviation of the noise current for the data bit of ' 1 ' [18]. The signal power and the noise power at the output of the LPF for the data bit of ' 1 ' are given by

$$
\begin{aligned}
& S=I_{1}{ }^{2} \\
& N=\sigma_{1}^{2}
\end{aligned}
$$

where $I_{1}$ is the normalized currents for the data bit ' 1 '. The noise power ratio (SNR) is given by

$$
S N R=S / N
$$

\section{PERFORMANCE ANALYSIS FOR PROPOSED SYSTEM USING SIMULATION}

In this section, simulations are performed with MATLAB on the basis of theoretical analysis on BER performances completed in previous section and some numerical results are shown and discussed. Here we assume that the receiver is designed with the PIN (Positive Intrinsic Negative) detector whose noise is dominated by the thermal noise at low receiving optical power and is independent of the average current $\left(\sigma_{0}=\sigma_{1}\right) \cdot[18]$

Figure 5 shows BER performances as a function of normalized threshold level at the SNR of $27 \mathrm{~dB}$ for asynchronous 2.5 Gbps/1.25 Gbps optical subscriber network with inverse RZ coded downstream and NRZ upstream re-modulation. When upstream data is ' 0 ', BER $P(1 / 0)$ is improved as the normalized threshold level increases. When upstream data is ' 1 ', the BER $P(0 / 1)$ is the sum of error probabilities for type a, type $\mathrm{b}$, type $\mathrm{c}$, type $\mathrm{d}$ and type e and it deteriorates as the normalized threshold level increases. Considering the signal power, it is seen that the BER $P(0 / 1)$ is more dominated by $P(1 / 0)$ than by error probabilities for type c, type $d$ and type e. As the total BER is the sum of $P(1 / 0)$ and $P(0 / 1)$, the BER performance is mainly determined by $P(1 / 0)$ and $P_{a}(0 / 1)$. Namely when the normalized threshold level is low, the total BER is dominated by $P(1 / 0)$. As the normalized threshold level increases, the total BER is dominated by $P_{a}(0 / 1)$ out of error probabilities for type a, type b, type c, type $\mathrm{d}$ and type e. So the receiver threshold level for the best BER performance is optimized at the near point of intersection between $P(1 / 0)$ and $P_{a}(0 / 1)$, the optimal normalized threshold level would be gained between 0 and $2 / 3$ which are the average normalized currents of the data ' 0 ' and type 'a' cases $\left(P_{a}(0 / 1)\right)$. because the total BER is limited by $P_{a}(0 / 1)$ as the normalized threshold level increasesWhen the SNR is $27 \mathrm{~dB}$, the best BER performance of $3 \times 10^{-16}$ is achieved at the normalized threshold level of $1 / 3$.

Figure 6 shows relationships among the best BER, the optimal normalized threshold level, and the SNR. When the SNR is lower than $25 \mathrm{~dB}$, the total BER is more dominated by $P(1 / 0)$ than $P_{a}(0 / 1)$. As the SNR increases more than $\sim 25 \mathrm{~dB}$, the total BER is more dominated by $P_{a}(0 / 1)$ than $P(1 / 0)$. Namely when the

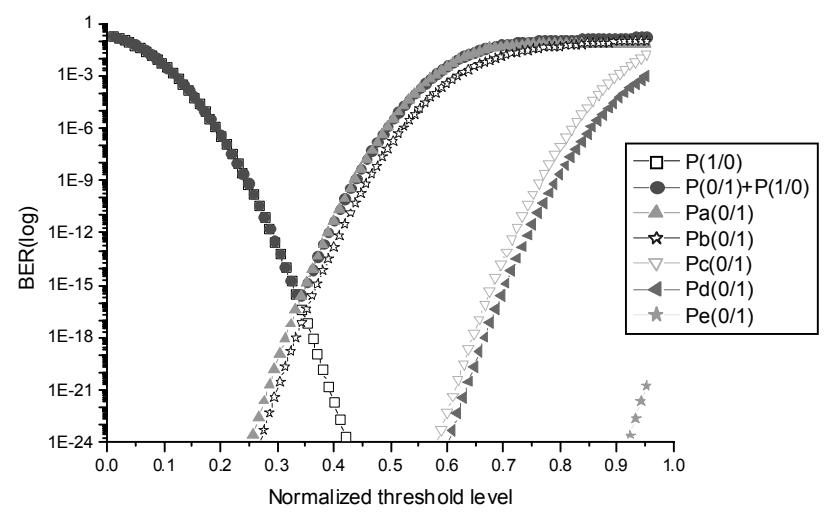

FIG. 5. BER performances as a function of normalized threshold level at the SNR of $27 \mathrm{~dB}$. 


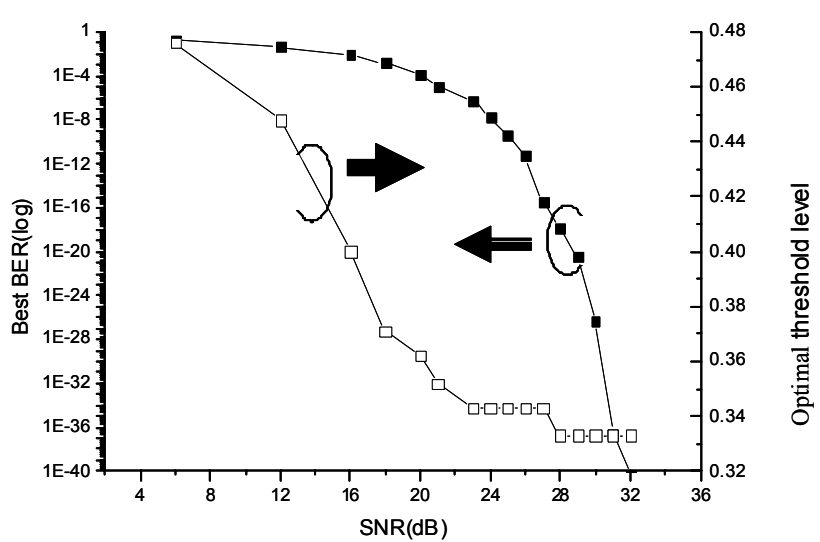

FIG. 6. Relationships among the best BER, the optimal normalized threshold level and the SNR.

SNR is low, the best BER is dominated by $P(1 / 0)$. As the SNR increases, the best BER is dominated by $P_{a}(0 / 1)$ out of error probabilities for type a, type b, type $\mathrm{c}$, type $\mathrm{d}$ and type e. We assume we are using the PIN detector whose noise is dominated by the thermal noise and is independent of the average current.[19] When the SNR increases, the best BER is limited by $P_{a}(0 / 1)$ and the optimal normalized threshold level comes to $1 / 3$, the mean between 0 and $2 / 3$. It is seen that the optimal normalized threshold level becomes saturated at $1 / 3$ as the SNR increases. The best BER performance can be gained by using the fixed normalized threshold level without controlling the threshold level of a receiver if the SNR attained is more than $25 \mathrm{~dB}$. Note that the optical threshold level Especially for obtaining the better BER than $10^{-9} 10^{-6}$ is between $0.33 \sim 0.35$. the needed SNR is larger than $\sim 25 \mathrm{~dB}$. So, This means that the receiver having the fixed threshold level of $1 / 3$ is very useful for real PON systems.

Figure 7 shows BER performances as a function of SNR in the cases of the fixed threshold level at $1 / 3$ and the conventional threshold level at 0.5 for asynchronous $2.5 \mathrm{Gbps} / 1.25 \mathrm{Gbps}$ optical subscriber network with inverse RZ coded downstream and NRZ upstream re-modulation. As the SNR increases more than $25 \mathrm{~dB}$, the BER is more dominated by $P_{a}(0 / 1)$ than $P(1 / 0)$ and the optimal normalized threshold level (TL) becomes saturated at $1 / 3$. As the SNR increases, BER performance can be improved more by using the fixed threshold level at $1 / 3$ than by the general receiver with threshold level of 0.5 . In the case of the fixed threshold level of $1 / 3$, the BER of $5.6 \times 10^{-10}$ is gained at the SNR of $25 \mathrm{~dB}$. In the case of the general receiver with threshold level of 0.5 , the BER of $1.8 \times 10^{-10}$ is gained at the SNR of $31 \mathrm{~dB}$. The threshold level is fixed optimized by adjusting the offset of an LA. The SNR necessary for obtaining BER $10^{-9}$ can be reduced by $\sim 5$ $\mathrm{dB}$ by fixing the normalized threshold level at $1 / 3$

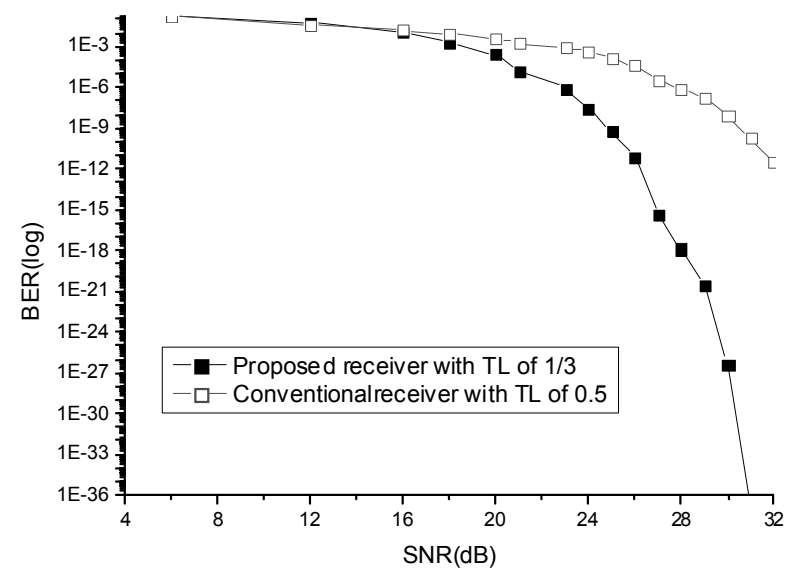

FIG. 7. BER performances as a function of SNR in the cases of the fixed threshold level at $1 / 3$ and the conventional threshold level at 0.5 .

instead of using the general receiver with threshold level of 0.5 . It means that the minimum receiving power of a receiver can be improved and a low power and low cost light source can be used in this proposed system.

\section{CONCLUSION}

In this paper, we have proposed the performance enhancing method optimization of asynchronous $2.5 \mathrm{Gbps} /$ $1.25 \mathrm{Gbps}$ optical subscriber network with inverse RZ coded downstream and NRZ upstream re-modulation by the threshold level control adjusting the threshold level of a limiting amplifier at a receiver. We have theoretically analyzed BER performance by modeling the occurrence of BER by simulation with MATLAB. The SNR necessary for obtaining BER $10^{-9}$ can be reduced by $\sim 5 \mathrm{~dB}$ by optimizing the normalized threshold level at $1 / 3$ instead of using the conventional receiver with threshold level of 0.5 .

The results have shown that the re-modulation scheme with inverse RZ coded downstream could be a useful technology for asynchronous optical access networks with asymmetric upstream and downstream data rates because the reduced minimum receiving power could replace a light source with a low power and low cost light source in an OLT.

\section{REFERENCES}

1. ITU-T Recommendation G.983.1, Broadband Optical Access Systems Based on PON, Oct. 1998.

2. M. D. Prycker, Asynchronous Transfer Mode: Solution for the Broadband ISDN, 3rd ed. (Prentice Hall PTR, USA, 1995). 
3. T. Pfeiffer, B. Deppisch, M. Kaiser, and R. Heidemann, "High speed optical network for asynchronous multiuser access applying periodic spectral coding of broadband sources," Electron. Lett. 33, 2141-2142 (1997).

4. M. S. Lee, B. T. Lee, J. D. Kim, and D. S. Lee, "Fabrication of 2.5 Gbps burst-mode receiver and its full compliance to GPON," J. Opt. Soc. Korea 12, 355-358 (2008).

5. N. J. Frigo, P. P. Iannone, P. D. Magill, T. E. Darcie, M. M. Downs, B. N. Desai, U. Koren, T. L. Koch, C. Dragone, H. M. Presby, and G. E. Bodeep, "A wavelength-division multiplexed passive optical network with cost-shared component," IEEE Photon. Technol. Lett. 6, 1365-1367 (1994).

6. S. Narikawa, H. Sanjoh, N. Sakurai, and K. Kumozaki, "Gbit-class transmission using SOA data rewriter for WDM-PON," IEICE Trans. Commun. E91-B, 399-408 (2008).

7. B. W. Kim, "RSOA-based wavelength-reuse gigabit WDM-PON," J. Opt. Soc. Korea 12, 337-345 (2008).

8. W. Hung, C.-K. Chan, L.-K. Chen, and F. Tong, "An optical network unit for WDM access networks with downstream DPSK and upstream re-modulated OOK date using injection-locked FP laser," IEEE Photon. Technol. Lett. 15, 1476-1478 (2003).

9. J. Prat, V. Polo, C. Bock, C. Arellano, and J. J. V. Olmos, "Full-duplex single fiber transmission using FSK downstream and IM remote upstream modulators for fiber-to-the-home," IEEE Photon. Technol. Lett. 17, 702-704 (2005).

10. N. Deng, C.-K. Chan, L.-K. Chen, and F. Tong, "Data remodulation on downstream OFSK signal for upstream transmission in WDM passive optical network," Electron.
Lett. 39, 1741-1743 (2003).

11. O. Akanbi, J. Yu, and G.-K. Chang, "A new scheme for bidirectional WDM-PON using upstream and downstream channels generated by optical carrier suppression and separation technique," IEEE Photon. Technol. Lett. 18, 340-342 (2006).

12. C.-H. Lee and S.-G. Mun, "WDM-PON based on wavelength locked Fabry-Perot LDs," J. Opt. Soc. Korea 12, 326-336 (2008).

13. B. K. Kim, H. Park, S. Park, and K. Kim, "Optical access network scheme with downstream Manchester coding and upstream NRZ re-modulation," Electron. Lett. 42, 484-485 (2006).

14. B. K. Kim, H. S. Chung, S. H. Chang, and S. J. Park, "Performance enhancement by threshold level control of a receiver in WDM-PON system with Manchester coded downstream and NRZ upstream re-modulation," IEICE Trans. Commun. E91-B, 2994-2997 (2008).

15. H. S. Chung, B. K. Kim, and K. Kim, "Effects of upstream bit rate on a wavelength-remodulated WDMPON based on Manchester or inverse-return-to-zero coding," ETRI Journal 30, 255-260 (2008).

16. G.-W. Lu, N. Deng, C.-K. Chan, and L.-K. Chen, "Use of down stream inverse-RZ signal for upstream data re-modulation in a WDM passive optical network," in Proc. Optical Fiber Communication Conference (Anaheim, CA, USA, Feb. 2005), paper OFI8.

17. J.-H. Lee, D.-H. Han, Y.-J. Lee, and B.-Y. Choi, "Optimization of $40 \mathrm{~Gb} / \mathrm{s}$ WDM systems using supergaussian RZ pulses," J. Opt. Soc. Korea 12, 226-231 (2008).

18. G. P. Agrawal, Fiber-optic Commication Systems, 2nd ed. (John Wiley \& Sons Inc., USA, 1997), pp. 170-173. 